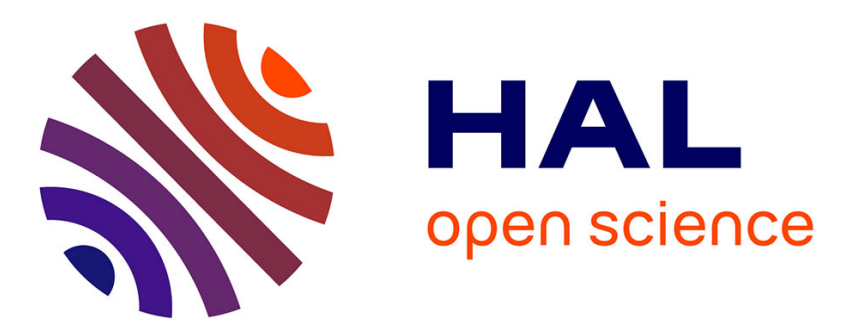

\title{
Evaporating droplet hologram simulation for digital in-line holography setup with divergent beam
}

Loï c Méè s, Nathalie Grosjean, Delphine Chareyron, Jean-Louis Mari é, Mozhdeh Seifi, Corinne Fournier

\section{> To cite this version:}

Loï c Méè s, Nathalie Grosjean, Delphine Chareyron, Jean-Louis Mari é, Mozhdeh Seifi, et al.. Evaporating droplet hologram simulation for digital in-line holography setup with divergent beam. Journal of the Optical Society of America. A Optics, Image Science, and Vision, 2013, 30 (10), pp.2021-2028. 10.1364/JOSAA.30.002021 . hal-00931483

\section{HAL Id: hal-00931483 https://hal.science/hal-00931483}

Submitted on 17 Jun 2014

HAL is a multi-disciplinary open access archive for the deposit and dissemination of scientific research documents, whether they are published or not. The documents may come from teaching and research institutions in France or abroad, or from public or private research centers.
L'archive ouverte pluridisciplinaire HAL, est destinée au dépôt et à la diffusion de documents scientifiques de niveau recherche, publiés ou non, émanant des établissements d'enseignement et de recherche français ou étrangers, des laboratoires publics ou privés. 


\title{
Evaporating Droplet Hologram Simulation for Digital In-line Holography set-up with Divergent Beam
}

\author{
Loïc Méès, ${ }^{1, *}$ Nathalie Grosjean, ${ }^{1}$ Delphine Chareyron, ${ }^{2}$ \\ Jean-Louis Marié, ${ }^{1}$ Mozhdeh Seifi, ${ }^{3}$ and Corinne Fournier ${ }^{3}$ \\ ${ }^{1}$ Laboratoire de Mécanique des Fluides et d'Acoustique (LMFA), UMR CNRS 5509 \\ Ecole Centrale de Lyon, Centre National de la Recherche Scientifique, \\ Université Claude Bernard Lyon 1, INSA de Lyon \\ 36 avenue Guy de Collongue, 69134 Ecully, France \\ ${ }^{2}$ Ecole Normale Supérieure de Lyon \\ 46 allée d'Italie, 69007 Lyon, France \\ ${ }^{3}$ Laboratoire Hubert Curien UMR5516 CNRS \\ Université Jean-Monnet \\ 18 rue $\operatorname{Pr}$ Benoit Lauras, F-42000, St Etienne, France
}

compiled: August 22, 2013

\begin{abstract}
Generalized Lorenz-Mie Theory (GLMT) for a multilayered sphere is used to simulate holograms produced by evaporating spherical droplets with refractive index gradient in the surrounding air/vapor mixture. Simulated holograms provide a physical interpretation of experimental holograms produced by evaporating Diethyl Ether droplets with diameter in the order of $50 \mu \mathrm{m}$ and recorded in a digital in-line holography configuration with a divergent beam. Refractive index gradients in the surrounding medium lead to a modification of the center part of the droplet holograms, where the first fringe is unusually bright. GLMT simulations reproduce well this modification, assuming an exponential decay of the refractive index from the droplet surface to infinity. The diverging beam effect is also considered. In both evaporating and non evaporating cases, an equivalence is found between Gaussian beam and plane wave illuminations, simply based on a magnification ratio to be applied to the droplets' parameters.
\end{abstract}

OCIS codes: $\quad$ (090.1995) holography, digital holography; (290.4020) scattering, Mie theory; (120.3940) instrumentation measurement and metrology, Metrological instrumentation.

http://dx.doi.org/10.1364/XX.99.099999

\section{Introduction}

Phase changes at droplet surface occur in many natural situations and industrial processes, in the atmosphere, in combustion chambers, in spray dryers and many other fluid mechanic engineering applications. Until recently, experimental studies of evaporating droplets were carried out from an Eulerian point of view, i.e. measurements were performed at a given point of the volume under study, providing local information on velocity or droplet size [1-3]. Such an approach is not suitable to capture the strong coupling between the evaporating droplets and their immediate environment, which requires a Lagrangian tracking of individual droplets providing both droplets' trajectories and size evolution.

Digital in-line holography (DIH) is a very promising optical technique to study the motion and the evolution of microscopic objects in $3 \mathrm{D}$ volumes, although it is

\footnotetext{
* Corresponding author: loic.mees@ec-lyon.fr
}

based on a rather simple experimental device. In association with a reconstruction algorithm based on an 'inverse problems' approach [4-6] and high speed cameras, DIH provides accurate location and size measurements, as required to track micro-droplets with rapid size evolution. In a previous paper [7], DIH has been used to study evaporating freon droplets in an isotropic and homogeneous turbulence. The droplets trajectories and their diameter evolution have been reconstructed from holograms by using an 'inverse problems' approach. This approach consists in minimizing the difference between an experimental droplet hologram and a parametric hologram formation model for an opaque spherical particle (a circular opaque disk, to be more specific).

It has been pointed out in [7] that the holograms are modified when evaporation takes place. In Fig. 1, a wake is visible in the vicinity of freon droplets, providing information on the Lagrangian relative motion of the air about the droplets. In addition, the central part of the holograms is abnormally bright compared to what 
is expected. This is the most problematic, because this over-brightness is not described by the standard hologram formation models. To get rid of this problem, an exclusion mask method is used in [7] to not take account for the central part of the hologram in the reconstruction process. The brightness excess is attributed to evaporation and the resulting refractive index gradient around the droplets, but there is no undisputable evidence of it. This paper proposes a physical explanation of this phenomenon, based on rigorous electromagnetic scattering calculations, assuming a perfect spherical symmetry. Experimental holograms of Diethyl Ether droplets that fulfill the spherical symmetry assumption are presented and Generalized Lorenz-Mie Theory for multilayered sphere is used to simulate holograms of droplets surrounded by a air/vapor mixture film showing a refractive index gradient.

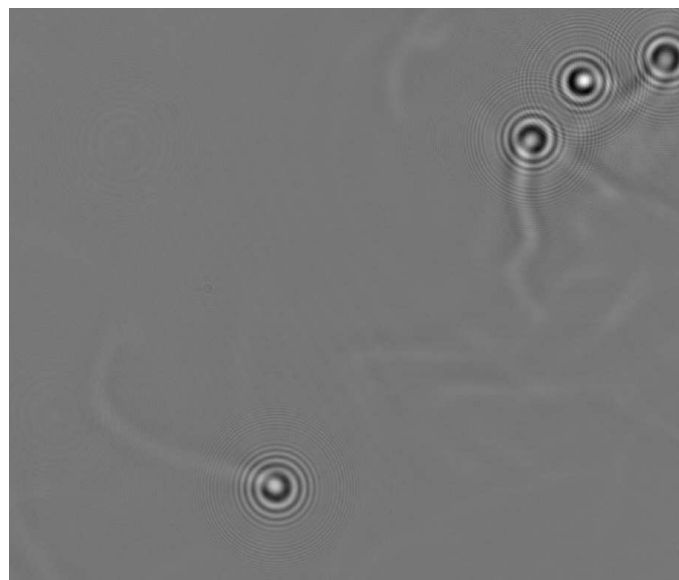

Fig. 1. Holographic image of freon droplets evaporating in a turbulent flow. The experiment set-up is detailed in [7].

Lorenz-Mie Theory [8-10] provides a quasi-analytic and rigourous solution to the scattering of a perfectly spherical, homogeneous and non-magnetic particle illuminated by a plane wave. During the last decades, this original 'theory' has been extended to different particle shapes and to non-uniform illumination under the name of Generalized Lorenz-Mie Theories (GLMT) [11, 12]. In this paper, GLMT for a multilayered sphere is applied to simulate holograms produced by spherical droplets surrounded by a refractive index gradient, under divergent beam illumination. Section 2 recalls the material needed to perform such computations. The main formulas required to perform the GLMT simulations, taken from different sources, are written under a uniform notation. Synthetic and experimental holograms produced by evaporating droplets are presented in section 3 . The effect of refractive index gradient in the surrounding medium is studied numerically and the effect of the beam divergence is considered for both evaporating and nonevaporating droplets. Section 4 is a conclusion.

\section{Generalized Lorenz-Mie Theory for a Multilayered sphere}

The problem of electromagnetic scattering from a multilayered sphere illuminated by a Gaussian beam can be solved rigorously in a Generalized Lorenz-Mie framework, using the Bromwich formulation [13]. The scattering problem is expressed and solved in spherical coordinates $(r, \theta, \varphi)$ in terms of scalar potentials from which all the electromagnetic field components are derived. The general solution to the scattering problem is written as the sum of two special solutions, the Tranverse-Electric (TE) for which the radial component of the electric field is nul $\left(E_{r}=0\right)$ and the Traverse-Magnetic (TM), for which the radial component of the magnetic field is nul $\left(H_{r}=0\right)$. The scattering particle is defined as an ensemble of $L$ concentric spherical layers of radius $r_{j}$ and a complex refractive index $n_{j}$ as shown in figure 2 . The surrounding medium is transparent, its refractive index $n_{0}$ is real. In this paper, all particle layers and the surrounding medium are assumed to be non magnetic. The origin of the cartesian and the spherical coordinate systems $((O, x, y, z)$ and $(O, r, \theta, \varphi))$ coincides with the particle center $O$. The particle is illuminated by a continous and monochromatic Gaussian beam, of wavelength $\lambda$, linearly polarized along $x$ direction. The beam waist is arbitrary located in $S$ of coordinates $\left(x_{0}, y_{0}, z_{0}\right)$ in $(O, x, y, z)$. The beam waist radius is denoted as $\omega_{0}$.

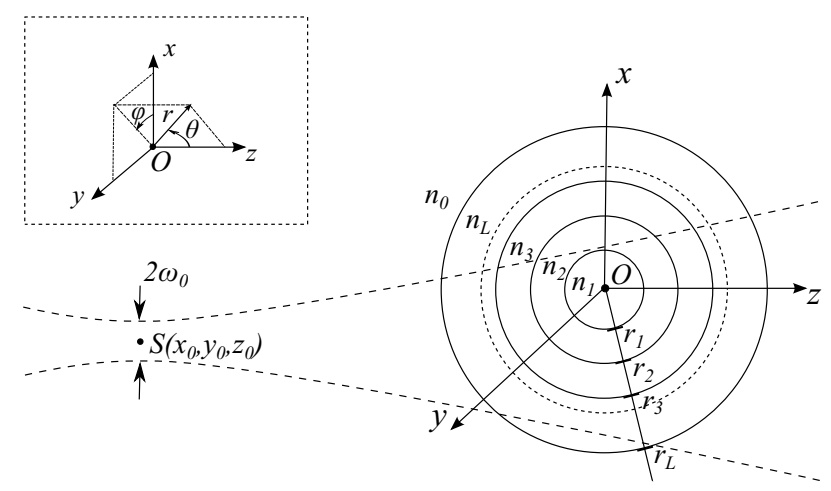

Fig. 2. Multilayered particle illuminated by a Gaussian beam.

In GLMT framework (as well as for LMT), incident field, scattered field and internal fields are expanded into series of spherical waves. Hovewer, the incident field components can be directly computed by using the first order (or 'Lowest' order) Davis approximation [14] expressed in spherical coordinates, omitting the timedependent harmonic term $\exp (i \omega t)$

$$
\begin{aligned}
E_{r}^{i}= & E_{0} \Psi_{0}\left[\cos \varphi \sin \theta\left(1-\frac{2 Q}{l} r \cos \theta\right)\right. \\
& \left.+\frac{2 Q}{l} x_{0} \cos \theta\right] \exp (K)
\end{aligned}
$$




$$
\begin{aligned}
E_{\theta}^{i}= & E_{0} \Psi_{0}\left[\cos \varphi\left(\cos \theta+\frac{2 Q}{l} r \sin ^{2} \theta\right)\right. \\
& \left.-\frac{2 Q}{l} x_{0} \sin \theta\right] \exp (K) \\
E_{\varphi}^{i}= & -E_{0} \Psi_{0} \sin \varphi \exp (K) \\
H_{r}^{i}= & H_{0} \Psi_{0}\left[\sin \varphi \sin \theta\left(1-\frac{2 Q}{l} r \cos \theta\right)\right. \\
& \left.+\frac{2 Q}{l} y_{0} \cos \theta\right] \exp (K) \\
H_{\theta}^{i}= & H_{0} \Psi_{0}\left[\sin \varphi\left(\cos \theta+\frac{2 Q}{l} r \sin ^{2} \theta\right)\right. \\
& \left.-\frac{2 Q}{l} y_{0} \sin \theta\right] \exp (K) \\
H_{\varphi}^{i}= & H_{0} \Psi_{0} \cos \varphi \exp (K)
\end{aligned}
$$

in which $l=k \omega_{0}^{2}$ is the diffraction length,

$$
\begin{aligned}
K= & -i k_{0}\left(r \cos \theta-z_{0}\right) \\
\Psi_{0}= & i Q \exp \left(-i Q \frac{r^{2} \sin ^{2} \theta}{\omega_{0}^{2}}\right) \exp \left(-i Q \frac{x_{0}^{2}+y_{0}^{2}}{\omega_{0}^{2}}\right) \\
& \times \exp \left[\frac{2 i Q}{\omega_{0}^{2}} r \sin \theta\left(x_{0} \cos \varphi+y_{0} \sin \varphi\right)\right] \\
Q= & \left(i+2 \frac{z-z_{0}}{k_{0} \omega_{0}^{2}}\right)^{-1}
\end{aligned}
$$

$k_{0}=2 \pi n_{0} / \lambda$ is the wave number in the surrounding medium.

The scattered fields components can be written as series of spherical waves as follows [15]

$$
\begin{aligned}
E_{r}^{s}= & -\frac{E_{0}}{k_{0}^{2} r^{2}} \sum_{n=1}^{n=\infty} \sum_{m=-n}^{m=+n} c_{n}^{p w} a_{n} g_{n, T M}^{m} n(n+1) \xi_{n}\left(k_{0} r\right) \\
& \times P_{n}^{|m|}(\cos \theta) \exp (i m \varphi) \\
E_{\theta}^{s}= & -\frac{E_{0}}{k_{0} r} \sum_{n=1}^{n=\infty} \sum_{m=-n}^{m=+n} c_{n}^{p w}\left[a_{n} g_{n, T M}^{m} \xi_{n}^{\prime}\left(k_{0} r\right) \tau_{n}^{|m|}(\cos \theta)\right. \\
& \left.+m b_{n} g_{n, T E}^{m} \xi_{n}\left(k_{0} r\right) \pi_{n}^{|m|}(\cos \theta)\right] \exp (i m \varphi) \\
E_{\varphi}^{s}= & -i \frac{E_{0}}{k_{0} r} \sum_{n=1}^{n=\infty} \sum_{m=-n}^{m=+n} c_{n}^{p w}\left[m a_{n} g_{n, T M}^{m} \xi_{n}^{\prime}\left(k_{0} r\right) \pi_{n}^{|m|}(\cos \theta)\right. \\
& \left.+b_{n} g_{n, T E}^{m} \xi_{n}\left(k_{0} r\right) \tau_{n}^{|m|}(\cos \theta)\right] \exp (i m \varphi) \\
H_{r}^{s}= & -\frac{H_{0}}{k_{0}^{2} r^{2}} \sum_{n=1}^{n=\infty} \sum_{m=-n}^{m=+n} c_{n}^{p w} b_{n} g_{n, T E}^{m} n(n+1) \xi_{n}\left(k_{0} r\right) \\
& \times P_{n}^{|m|}(\cos \theta) \exp (i m \varphi) \\
H_{\theta}^{s}= & -\frac{H_{0}}{k_{0} r} \sum_{n=1}^{n=\infty} \sum_{m=-n}^{m=+n} c_{n}^{p w}\left[m a_{n} g_{n, T M}^{m} \xi_{n}\left(k_{0} r\right) \pi_{n}^{|m|}(\cos \theta)\right. \\
& \left.-b_{n} g_{n, T E}^{m} \xi_{n}^{\prime}\left(k_{0} r\right) \tau_{n}^{|m|}(\cos \theta)\right] \exp (i m \varphi)
\end{aligned}
$$

$$
\begin{aligned}
H_{\varphi}^{s}= & i \frac{H_{0}}{k_{0} r} \sum_{n=1}^{n=\infty} \sum_{m=-n}^{m=+n} c_{n}^{p w}\left[a_{n} g_{n, T M}^{m} \xi_{n}\left(k_{0} r\right) \tau_{n}^{|m|}(\cos \theta)\right. \\
& \left.-m b_{n} g_{n, T E}^{m} \xi_{n}^{\prime}\left(k_{0} r\right) \pi_{n}^{|m|}(\cos \theta)\right] \exp (i m \varphi)
\end{aligned}
$$

where $a_{n}$ and $b_{n}$ are the scattering coefficients, $g_{n, T E}^{m}$ and $g_{n, T M}^{m}$ are the beam shape coefficients (BSPs) and $c_{n}^{p w}$ is defined as

$$
c_{n}^{p w}=(-i)^{n} \frac{2 n+1}{n(n+1)}
$$

$\xi_{n}(k r)$ is the Riccati-Bessel function based on the spherical Bessel function of the fourth kind $\Psi_{n}^{(4)}$ and the Hankel function of second kind $H_{n+\frac{1}{2}}^{(2)}$ by

$$
\xi_{n}(k r)=k r \Psi_{n}^{(4)}(k r)=\sqrt{\frac{\pi k r}{2}} H_{n+\frac{1}{2}}^{(2)}(k r)
$$

$\xi_{n}^{\prime}(k r)$ its derivative and the generalized Legendre function $\tau_{n}^{m}(\cos \theta)$ and $\pi_{n}^{m}(\cos \theta)$ are defined from the associated Legendre polynomials $P_{n}^{m}$ by

$$
\begin{aligned}
\tau_{n}^{m}(\cos \theta) & =\frac{\mathrm{d}}{\mathrm{d} \theta} P_{n}^{m}(\cos \theta) \\
\pi_{n}^{m}(\cos \theta) & =\frac{P_{n}^{m}(\cos \theta)}{\sin \theta}
\end{aligned}
$$

The most efficient way to compute the BSPs is the use of the localized approximation $[12,16]$, leading to the following expressions :

$$
\begin{aligned}
{\left[\begin{array}{c}
g_{n, T M}^{m} \\
g_{n, T E}^{m}
\end{array}\right]=} & \frac{1}{2\left(1+2 i z_{0}^{+}\right)} \exp \left(\frac{i z_{0}^{+}}{s^{2}}-\frac{x_{0}^{+^{2}}+y_{0}^{+2}}{1+2 i z_{0}^{+}}\right) \\
& \times \exp \left(-\frac{\left(n+\frac{1}{2}\right)^{2} s^{2}}{1+2 i z_{0}^{+}}\right) \\
& \times R_{n}^{m}(-i)^{|m|}\left[\begin{array}{c}
i F_{n, T M}^{m} \\
F_{n, T E}^{m}
\end{array}\right]
\end{aligned}
$$

where

$$
\begin{gathered}
R_{n}^{m}=\left(\frac{2}{2 n+1}\right)^{|m|-1},|m| \geq 1 \\
R_{n}^{0}=\frac{2 n(2 n+1)}{2 n+1} \\
{\left[\begin{array}{c}
F_{n, T M}^{0} \\
F_{n, T E}^{0}
\end{array}\right]=\left[\begin{array}{c}
2 x_{0}^{+} \\
2 i y_{0}^{+}
\end{array}\right] \sum_{j=0}^{\infty} a^{2 j+1} \frac{X_{-}^{j} X_{+}^{j}}{j !(j+1) !}}
\end{gathered}
$$


with

$$
\begin{aligned}
& {\left[\begin{array}{c}
F_{n, T M}^{m} \\
F_{n, T E}^{m}
\end{array}\right]=a^{m-1} \frac{X_{-}^{m-1}}{(m-1) !}+\sum_{j=m}^{\infty} a^{2 j-m+1} \frac{X_{-}^{j} X_{+}^{j-m}}{j !(j-m) !}} \\
& \times\left[\begin{array}{l}
\frac{X_{+}}{j-m+1}+\frac{X_{-}}{j+1} \\
\frac{X_{+}}{j-m+1}-\frac{X_{-}}{j+1}
\end{array}\right], m>0 \\
& {\left[\begin{array}{c}
F_{n, T M}^{m} \\
-F_{n, T E}^{m}
\end{array}\right]=a^{-1-m} \frac{X_{-}^{-1-m}}{(-1-m) !}+\sum_{j=-m}^{\infty} a^{2 j+m+1} \frac{X_{-}^{j+m} X_{+}^{j}}{j !(j+m) !}} \\
& \times\left[\frac{\frac{X_{-}}{j+m+1}+\frac{X_{+}}{j+1}}{\frac{X_{-}}{j+m+1}-\frac{X_{+}}{j+1}}\right], m<0
\end{aligned}
$$

where

$$
\begin{gathered}
a=\frac{(n+1 / 2) s}{1+2 i z_{0}^{+}} \\
X_{-}=x_{0}^{+}-i y_{0}^{+}, \quad X_{+}=x_{0}^{+}+i y_{0}^{+}
\end{gathered}
$$

and

$$
x_{0}^{+}=\frac{x_{0}}{\omega_{0}}, \quad y_{0}^{+}=\frac{y_{0}}{\omega_{0}}, \quad z_{0}^{+}=\frac{z_{0}}{k_{0} \omega_{0}^{2}}
$$

In case of a single homogeneous sphere (for both LMT and GLMT), the scattering coefficients $a_{n}$ and $b_{n}$ are deduced from the boundary conditions at the sphere surface. In case of a multilayered sphere boundary conditions are considered at each layer surface, leading to a recursive determination of the coefficients [13] following

$$
\begin{aligned}
a_{n} & =\frac{\psi_{n}\left(x_{L}^{\prime}\right) H_{n}^{a}\left(x_{L}\right)-m_{L} \psi_{n}^{\prime}\left(x_{L}^{\prime}\right)}{\xi_{n}\left(x_{L}^{\prime}\right) H_{n}^{a}\left(x_{L}\right)-m_{L} \xi_{n}^{\prime}\left(x_{L}^{\prime}\right)} \\
b_{n} & =\frac{m_{L} \psi_{n}\left(x_{L}^{\prime}\right) H_{n}^{b}\left(x_{L}\right)-\psi_{n}^{\prime}\left(x_{L}^{\prime}\right)}{m_{L} \xi_{n}\left(x_{L}^{\prime}\right) H_{n}^{b}\left(x_{L}\right)-\xi_{n}^{\prime}\left(x_{L}^{\prime}\right)}
\end{aligned}
$$

where $x_{j}=k_{j} r_{j}, x_{j}^{\prime}=k_{j+1} r_{j}, x_{L}^{\prime}=k_{0} r_{L} . \quad k_{j}$ and $m_{j}=n_{j} / n_{0}$ designates the wavenumber and the relative refractive index in layer $j . \psi_{n}$ is the RiccatiBessel function based on the spherical Bessel function of the first kind $\Psi_{n}^{(1)}$ and the Bessel fonction of the first kind $J_{n}$ following

$$
\psi_{n}(k r)=k r \Psi_{n}^{(1)}(k r)=\sqrt{\frac{\pi k r}{2}} J_{n+\frac{1}{2}}(k r)
$$

and $\psi_{n}^{\prime}$ its derivative. The terms $H_{n}^{a}\left(x_{L}\right)$ and $H_{n}^{b}\left(x_{L}\right)$ in relation (18) are calculated recursively following

$$
\begin{aligned}
& H_{n}^{a}\left(x_{j}\right)=H_{n}^{b}\left(x_{j}\right)=\frac{\psi_{n}^{\prime}\left(x_{1}\right)}{\psi_{n}\left(x_{1}\right)} \\
& H_{n}^{a}\left(x_{j}\right)=\frac{\psi_{n}^{\prime}\left(x_{j}\right)-R_{j n}^{a} \chi_{n}^{\prime}\left(x_{j}\right)}{\psi_{n}\left(x_{j}\right)-R_{j n}^{a} \chi_{n}\left(x_{j}\right)} \\
& H_{n}^{b}\left(x_{j}\right)=\frac{\psi_{n}^{\prime}\left(x_{j}\right)-R_{j n}^{b} \chi_{n}^{\prime}\left(x_{j}\right)}{\psi_{n}\left(x_{j}\right)-R_{j n}^{b} \chi_{n}\left(x_{j}\right)}
\end{aligned}
$$

$$
\begin{aligned}
& R_{1 n}^{a}=R_{1 n}^{b}=0 \\
& R_{2 n}^{a}=\frac{m_{2} \psi_{n}\left(x_{1}^{\prime}\right) \psi_{n}^{\prime}\left(x_{1}\right)-m_{1} \psi_{n}^{\prime}\left(x_{1}^{\prime}\right) \psi_{n}\left(x_{1}\right)}{m_{2} \chi_{n}\left(x_{1}^{\prime}\right) \psi_{n}^{\prime}\left(x_{1}\right)-m_{1} \chi_{n}^{\prime}\left(x_{1}^{\prime}\right) \psi_{n}\left(x_{1}\right)} \\
& R_{2 n}^{b}=\frac{m_{1} \psi_{n}\left(x_{1}^{\prime}\right) \psi_{n}^{\prime}\left(x_{1}\right)-m_{2} \psi_{n}^{\prime}\left(x_{1}^{\prime}\right) \psi_{n}\left(x_{1}\right)}{m_{1} \chi_{n}\left(x_{1}^{\prime}\right) \psi_{n}^{\prime}\left(x_{1}\right)-m_{2} \chi_{n}^{\prime}\left(x_{1}^{\prime}\right) \psi_{n}\left(x_{1}\right)}
\end{aligned}
$$

and for $j>2$

$$
\begin{aligned}
R_{j n}^{a} & =\frac{m_{j} \psi_{n}\left(x_{j-1}^{\prime}\right) H_{n}^{a}\left(x_{j-1}\right)-m_{j-1} \psi_{n}^{\prime}\left(x_{j-1}^{\prime}\right)}{m_{j} \chi_{n}\left(x_{j-1}^{\prime}\right) H_{n}^{a}\left(x_{j-1}\right)-m_{j-1} \chi_{n}^{\prime}\left(x_{j-1}^{\prime}\right)} \\
R_{j n}^{b} & =\frac{m_{j-1} \psi_{n}\left(x_{j-1}^{\prime}\right) H_{n}^{b}\left(x_{j-1}\right)-m_{j} \psi_{n}^{\prime}\left(x_{j-1}^{\prime}\right)}{m_{j-1} \chi_{n}\left(x_{j-1}^{\prime}\right) H_{n}^{b}\left(x_{j-1}\right)-m_{j} \chi_{n}^{\prime}\left(x_{j-1}^{\prime}\right)}
\end{aligned}
$$

in which $\chi_{n}$ designates the Riccati-Bessel function based on the spherical Bessel function of the second kind $\Psi_{n}^{(2)}$ and the Bessel fonction of the second kind $Y_{n}$ following

$$
\chi_{n}(k r)=k r \Psi_{n}^{(2)}(k r)=\sqrt{\frac{\pi k r}{2}} Y_{n+\frac{1}{2}}(k r)
$$

and $\chi_{n}^{\prime}$ its derivative.

To simulate digital in-line holograms, incident fields and scattered fields are computed onto the sensor plane ( $z=z_{E}=$ constant), following (1)-(6) and (7)-(12) respectively. The total field is then computed by summation, that is $\mathbf{E}^{t}=\mathbf{E}^{i}+\mathbf{H}^{s}$ and $\mathbf{H}^{t}=\mathbf{H}^{i}+\mathbf{H}^{s}$. Intensities and propagation direction are given by the Poynting vector:

$$
\mathbf{S}^{t}=\frac{1}{2} \operatorname{Re}\left[\mathbf{E}^{t} \times \mathbf{H}^{t^{*}}\right]
$$

The recorded intensity is then the projection of $\mathbf{S}^{t}$ on direction $z$, perpendicular to the sensor, that is

$$
\begin{aligned}
S_{\perp}^{t}= & \frac{1}{2} \operatorname{Re}\left[\cos \theta\left(E_{\theta}^{t} H_{\varphi}^{t^{*}}-E_{\varphi}^{t} H_{\theta}^{t^{*}}\right)\right. \\
& \left.-\sin \theta\left(E_{\varphi}^{t} H_{r}^{t^{*}}-E_{r}^{t} H_{\varphi}^{t^{*}}\right)\right]
\end{aligned}
$$

In the following, a normalized intensity $I_{N}$ will be used to facilitate comparisons. This normalized intensity is defined relatively to the non-perturbed incident intensity $S_{\perp}^{i}$ as follows

$$
I_{N}=\frac{S_{\perp}-S_{\perp}^{i}}{S_{\perp}^{i}}
$$

where

$$
\begin{aligned}
S_{\perp}^{i}= & \frac{1}{2} \operatorname{Re}\left[\cos \theta\left(E_{\theta}^{i} H_{\varphi}^{i^{*}}-E_{\varphi}^{i} H_{\theta}^{i^{*}}\right)\right. \\
& \left.-\sin \theta\left(E_{\varphi}^{i} H_{r}^{i^{*}}-E_{r}^{i} H_{\varphi}^{i^{*}}\right)\right]
\end{aligned}
$$




\section{Evaporating droplet holograms with divergent beam illumination}

\section{A. Experimental set-up}

Holograms presented in [7] show unusually bright central fringes and non-symmetric wakes which are elongated because of the relative velocities between the freon drops and the turbulent air-flow. To find a physical interpretation of the holographic pattern central parts, a new experiment has been conducted on free falling Diethyl Ether droplets in still air. In this case, the relative velocities are small enough to fulfill the symmetry contrainst imposed by GLMT calculations. Ether droplets are illuminated by a continuous divergent laser beam (wavelenght $\lambda=532 \mathrm{~nm}$ produced by a neodymium-doped yttrium orthovanadate $\left(N d: Y_{V} O_{4}\right)$ laser in a digital in line holography configuration, as shown in Fig. 3. The laser beam is focused in $S$ by means of a $25 \mathrm{~mm}$ focal length lens. The distance between the object (Ether droplets or calibration target) and the CMOS sensor is $z_{E}=360 \mathrm{~mm}$. The distance $z_{S}$ between the point source (or beam waist) and the object is about $555 \mathrm{~mm}$. As an example, a droplet hologram profile is ploted in figure 3 , showing the unsually bright central fringe, to be later discussed in this paper.

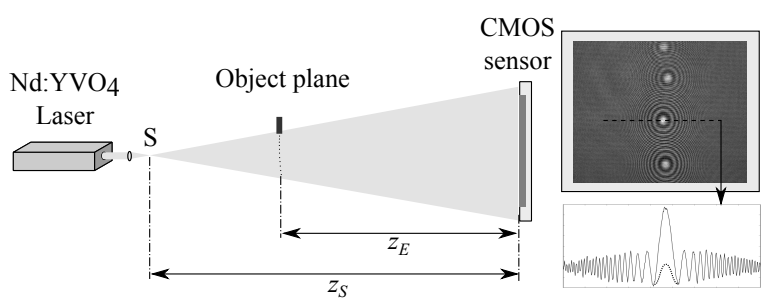

Fig. 3. Experimental set-up. A divergent beam is used to record evaporating Diethyl Ether droplet holograms.

\section{B. Divergent beam effect for non evaporating droplet}

The use of a divergent beam leads to a magnification of the holograms $[17,18]$ for both object size and reconstruction distance, with a magnification ratio

$$
G=\frac{z_{S}}{z_{S}-z_{E}}
$$

The hologram produced by a drop of radius $r_{D}$ and recorded at a distance $z_{E}$ in the divergent beam configuration of figure 3 is then equivalent to the hologram produced by a drop of radius $G r_{D}$, illuminated by a plane wave and recorded at a distance $G z_{E}$. The magnification ratio has been measured by using a calibration target and a standard reconstruction process, for different $z_{E}$ positions at constant $z_{S}$ (moving target along $z$ ), leading to $G=2.86$ for $z_{E}=360 \mathrm{~mm}$ and a refinement of the point source position measurement $z_{S}=553.5 \mathrm{~mm}$. The magnification introduced by the beam divergence can be demonstrated in the Fresnel integral framework [17, 18].
The equivalence is verified in the GLMT simulations presented Fig. 4. This figure shows sections of the holograms, taking advantage of their circular symetry. These holograms are produced at a distance $z_{E}=360 \mathrm{~mm}$ by homogeneous spherical particles of radius $r_{D}=20 \mu \mathrm{m}$ and $r_{D}=50 \mu \mathrm{m}$, illuminated by a Gaussian beam of beam waist radius $\omega_{0}=1 \mu \mathrm{m}$, wavelength $\lambda=532 \mathrm{~nm}$ and focused on $\left(x_{0}, y_{0}, z_{0}\right)=\left(0,0,-\left(z_{S}-z_{E}\right)\right)$. These holograms perfectly fit the holograms produced at a distance $G z_{E}=1029.6 \mathrm{~mm}$ by spherical droplets of radius $G r_{D}=57.2 \mu \mathrm{m}$ and $G r_{D}=143 \mu \mathrm{m}$ respectively, illuminated by a plane wave. In this example, the spherical particles contain only one layer of radius $r_{1}=r_{L}=r_{D}$. The refractive index of liquid Ether is set to $n_{1}=(1.35-0 i)$ and the surrounding medium refractive index is set to $n_{0}=1$.

\section{C. Evaporating droplet holograms}

Evaporating droplets are characterized by the presence of a refractive index gradient inside the droplet (related to temperature gradient) and by the presence of a air/vapor mixture film all around it. The internal refractive index gradient can be considered in GLMT simulation by considering a large number of layers with a nearly continuous refractive index evolution [19]. GLMT simulations (not presented here) show that such an internal gradient does not affect the droplet holograms in the present configuration (at large distance) and will be not considered in the following.

The surrounding medium is also characterized by a refractive index gradient, due to the evolution of both temperature and Ether vapor concentration from the droplet surface to surrounding air. Following [20], the refractive index gradient near the evaporating droplet surface can be described by an exponential decay. Assuming a spherical symmetry, evaporating droplets will be defined as multilayered spheres with a first layer corresponding to the liquid droplet of radius $r_{1}=r_{D}$ and refractive index $n_{1}=(1.35-0 i)$ surrounded by a large number of layers with regularly spacing radii $r_{j}$ and refractive indices $n_{j}$ following an exponential decay from the droplet surface to infinity of the form

$$
n(r)=n_{0}+n_{s} \exp \left(-\frac{r-r_{D}}{\sigma}\right)
$$

where $n_{0}$ is the surrounding air refractive index (far from the droplet), $n_{s}$ the refractive index deviation from $n_{0}$ at the droplet surface (in the gas phase) and $\sigma$ is a width parameter. The spacing between the radii $r_{j}$ and the refractive indices $n_{j}$ must be small enough to describe accurately the continuous evolution of relation (30). The total number of layers $L$ required and the external radius $r_{L}$ of the particle (droplet and surrounded film) are determined from tests of convergence. In the following, $L$ is set to 200 and $r_{L}$ to $6 \sigma$.

Figure 5.b shows synthetic holograms computed by GLMT for $r_{D}=20 \mu \mathrm{m}$ radius droplets surrounded by re- 
fractive index gradients with constant width parameter $\sigma=80 \mu m$ and various refractive index deviation $n_{s}$ as shown in figure 5.a. The droplets are illuminated by the divergent beam as defined above $\left(\omega_{0}=1 \mu \mathrm{m}, \lambda=532 \mathrm{~nm}\right.$ and $\left.z_{0}=-\left(z_{S}-z_{E}\right)=-193.5 \mathrm{~mm}\right)$ and the distance between the droplet and the sensor is $z_{E}=360 \mathrm{~mm}$. The surrounding air refractive index (far from the droplets) is $n_{0}=1$. The case $n_{s}=0$ corresponds to a droplet immersed in a homogeneous medium, that is a nonevaporating droplet. The corresponding hologram is used as a reference. It shows a standard pattern for the large recording distance considered here, with a central fringe amplitude (in $x=0$ ) close to the average intensity of the whole hologram, which is about zero with the normalization adopted here. In the presence of refractive index gradient $\left(n_{s} \neq 0\right)$, the central part of the holograms is modified. The central fringe amplitude increases with increasing $n_{s}$, showing the same behavior as observed experimentaly. For $n_{s} \leq 10^{-6}$, the hologram is almost unaffected by the refractive index gradient. For $n_{s}=5 \times 10^{-6}$ and $n_{s}=10^{-5}$, only the central fringe is affected. For greater values of $n_{s}$, the second fringe of the hologram is also modified by the presence of refractive index gradient.

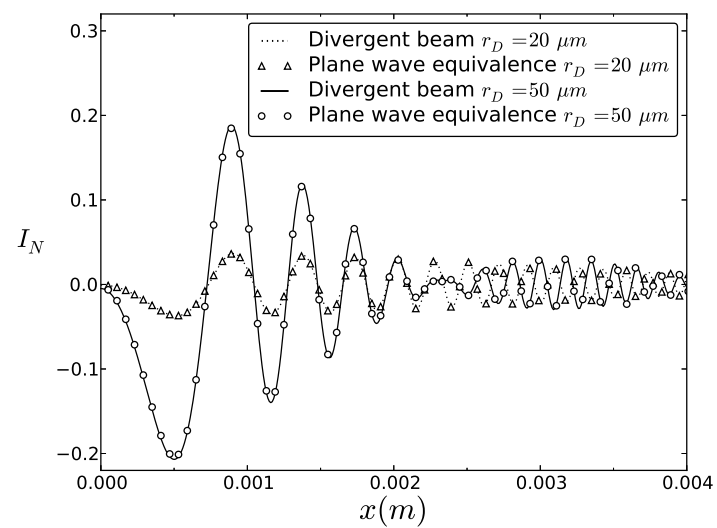

Fig. 4. Comparison between holograms produced under focused beam and plane wave illumination. The plane wave equivalence consists in applying the magnification ratio $G$ defined in Eq. 29 on both droplet radius and recording distance.

Figure 6.b shows synthetic holograms computed for $r_{D}=20 \mu \mathrm{m}$ radius droplets surrounded by refractive index gradients with constant $n_{s}=5 \times 10^{-5}$ and different width parameter $\sigma$, from 0 (no gradient) to $320 \mu \mathrm{m}$ (16 times $r_{D}$ ), with other parameters unchanged with respect to Fig. 5. The corresponding refractive index profiles are plotted in figure 6.a. The central fringe amplitude increases with increasing $\sigma$ but it reaches a maximum for $\sigma$ near about $200 \mu \mathrm{m}$ (not represented) and then slightly decreases for greater $\sigma$. The second fringe is increasingly affected for increasing $\sigma$ as it was for $n_{s}$ increasing in the previous figure. The next fringes am- plitudes are nearly unaffected but it must be noted that for $\sigma \geq 160 \mu \mathrm{m}$ the presence of a surrounding gradient induces a slight translation of these fringes. The results of Figs. 5 and 6 show that only the three first fringes are notably affected by the refractive index gradient in the case of a $r_{D}=20 \mu \mathrm{m}$ radius droplet and in the limit of $n_{s} \leq 10^{-4}$ and $\sigma \leq 4 r_{D}$. Then, a masking method, similar to the one used in [7], can be used to extract the location and diameter of the evaporating droplets by using an 'inverse problems' approach. After detecting the holographic signature of an object, its central part (first three fringes) can be excluded from the analysis and the rest of the pattern can be compared to synthetic holograms computed with a simple analytical model (opaque disk and Fresnel integral propagation) which does not take the surrounding gradient into account.

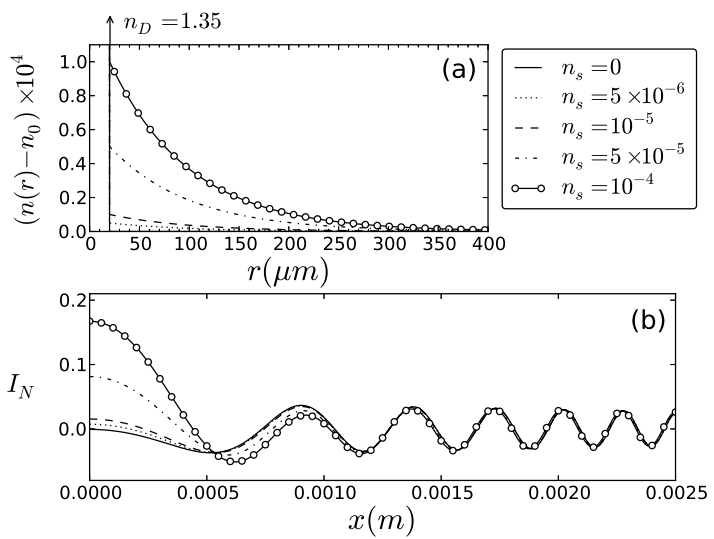

Fig. 5. Evaporating droplet holograms for varying refractive index deviation at droplet surface. The droplet is illuminated by a Gaussian beam $\left(\omega_{0}=1 \mu \mathrm{m}, \lambda=532 \mathrm{~nm}\right.$ and $\left.z_{0}=-\left(z_{S}-z_{E}\right)=-193.5 \mathrm{~mm}\right)$. The droplet radius is $r_{D}=20 \mu \mathrm{m}$, refractive index decreases exponentially in the surrounding medium following Eq. 30 with variable $n_{s}$ and a constant decay parameter $\sigma=80 \mu \mathrm{m}$.

Figure 7 shows the effect of the refractive index gradient on holograms, for different droplet sizes. In this figure, the droplet radius varies from 10 to $30 \mu \mathrm{m}$ and the resulting holograms, with and without gradients, are compared. For each droplet size, the refractive index gradient is defined by a constant refractive index deviation at the droplet surface $n_{s}=5 \times 10^{-5}$ and by a width parameter varying proportionnally to the droplet radius, that is $\sigma=4 r_{D}$. Other parameters remain unchanged with respect to the previous figures. It can be seen here that the modification of the holograms is still mainly focused on the first fringes. However, other fringes are progressively modified by the gradient when the droplet size increases. This appears more clearly in Fig. 8 where larger droplets of radius $r_{D}=50 \mu \mathrm{m}$ and $r_{D}=100 \mu \mathrm{m}$ are considered, with the same other parameters as in Fig. 7. In both cases, the first two fringes are clearly affected. All the next fringes are unaffected in terms of 

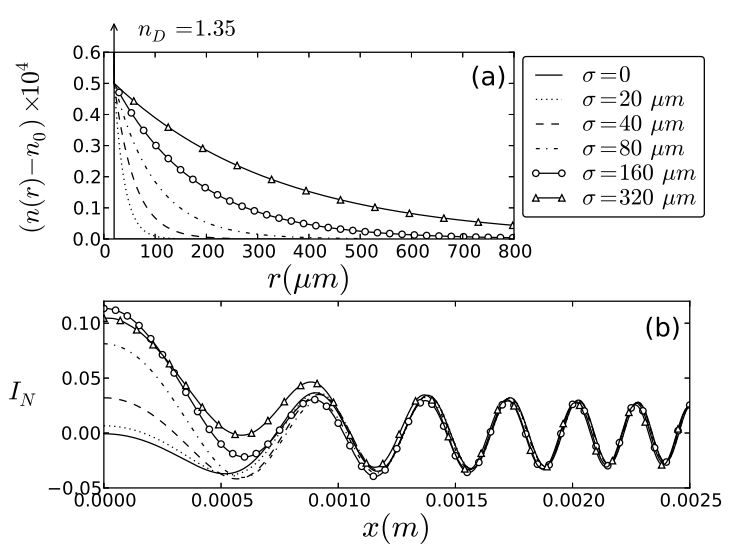

Fig. 6. Evaporating droplet holograms for varying decay parameter $\sigma$. The droplet is illuminated by a Gaussian beam $\left(\omega_{0}=1 \mu \mathrm{m}, \lambda=532 \mathrm{~nm}\right.$ and $z_{0}=-\left(z_{S}-z_{E}\right)=$ $-193.5 \mathrm{~mm})$. The droplet radius is $r_{D}=20 \mu \mathrm{m}$ and the refractive index decreases exponentially in the surrounding medium following Eq. 30 with variable $\sigma$ from 0 to $320 \mu \mathrm{m}$ and a constant refractive index deviation at droplet surface $n_{s}=5 \times 10^{-5}$.

amplitude but they are clearly shifted as a consequence of the surrounded gradient, including the fringes beyond the first zero of the Airy function. Then, in this case, one can expect some bias in the estimation of $z$ and $r_{D}$ using an exclusion mask.

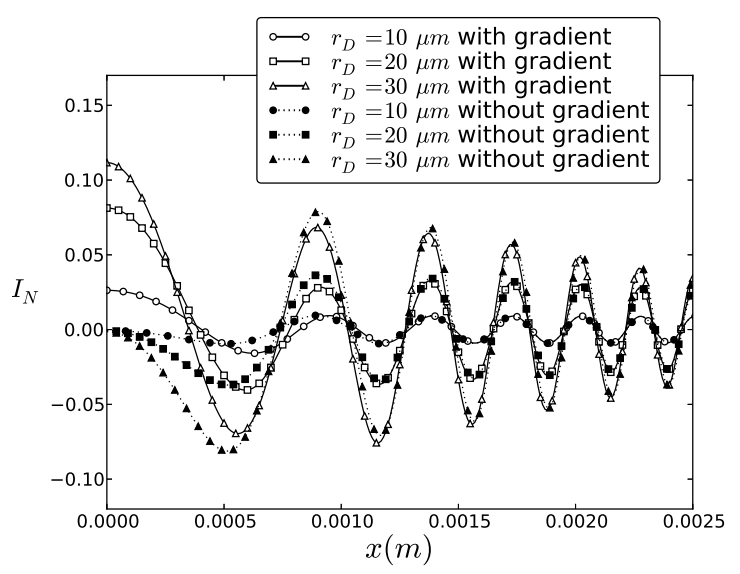

Fig. 7. Effect of the refractive index gradient on holograms for different droplet sizes. The holograms are produced by different droplets of radius $r_{D}=10 \mu \mathrm{m}, r_{D}=20 \mu \mathrm{m}$ and $r_{D}=30 \mu \mathrm{m}$, with and wihout surrounding refractive index gradients, which are defined by a constant deviation at droplet surface $n_{s}=5 \times 10^{-5}$ and a width parameter proportional to the droplet radius $\sigma=4 r_{D}$.

This work opens a way to a complete characterization of evaporating droplets, including the surrounding refractive index gradient. The inversion of experimental data is out of this paper scope, but the hologram simulations discussed above suggest that a two step in-

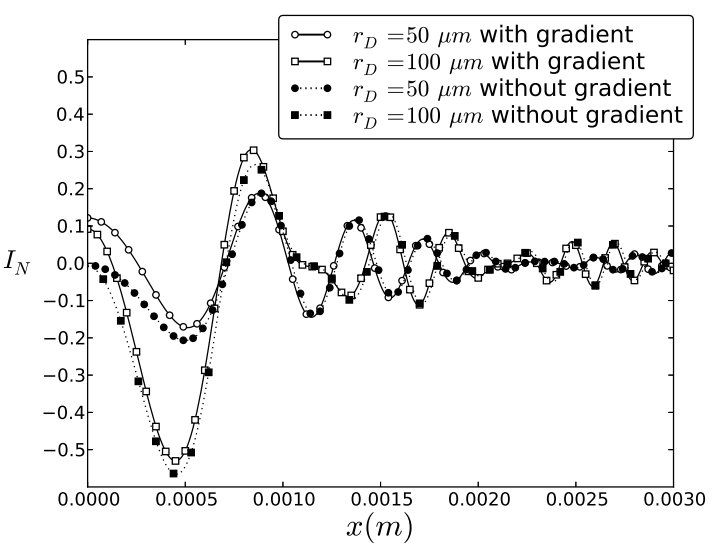

Fig. 8. Effect of the surrounding refractive index gradient on holograms for different droplet sizes. The holograms are produced by droplets of radius $r_{D}=50 \mu \mathrm{m}$ and $r_{D}=100 \mu \mathrm{m}$, with and wihout surrounding refractive index gradients, which are defined by a constant deviation at droplet surface $n_{s}=5 \times 10^{-5}$ and a width parameter proportional to the droplet radius $\sigma=4 r_{D}$.

version procedure could be used. To examplify the potential of this approach, figure 9 shows an experimental hologram profile of an evaporating Ether droplet. This profile results from an angular averaging of a $2 \mathrm{D}$ hologram recorded in the experimental conditions described in section 3.A. A masking method is first apply to determine the droplet radius $r_{D}$ and its location $z$ : the difference between the synthetic hologram and the experimental one is minimized, considering only $x$ ranging from $1.5 \mathrm{~mm}$ to $6 \mathrm{~mm}$ (dotted lines in Fig. 9), excluding the first three fringes. The first step leads to $r_{D}=21 \mu \mathrm{m}$ and $z=360.426 \mathrm{~mm}$. The synthetic hologram of the corresponding droplet (without surrounding gradient) is shown in the figure. Then, a surrounding refractive index with exponential decay is considered in the hologram model. A second minimization procedure is applied with fixed $r_{D}$ and $z$, to determine the surface refractive index deviation $n_{s}=8.5 \times 10^{-5}$ and the decay parameter $\sigma=80 \mu \mathrm{m}$. The evaporating droplet synthetic hologram almost agrees with the experiments, excepted for a significant deviation in the second and third fringes amplitude. This departure is probably due to a significant deviation from the ideal exponential decay considered in simulations. The $n_{s}$ value deduced from the experimental hologram is of the same order of magnitude as the one obtained in [20] $\left(n_{s}=1.5 \times 10^{-4}\right)$ for large Ether droplets $(\sim 2 \mathrm{~mm})$ suspended on a stainless steel fiber with thermal conductivity. It is even closer to the value obtained in [21] $\left(n_{s}=1.2 \times 10^{-4}\right)$ for Ether droplet of about $1.5 \mathrm{~mm}$ suspended on a plastic fiber with low thermal conductivity. 


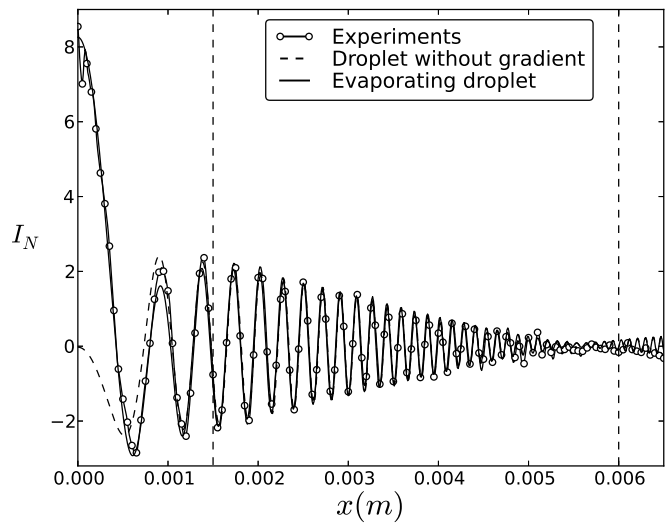

Fig. 9. Experimental hologram compared to computed holograms, with and wihout surrounding refractive index gradient. For both evaporating and non evaporating case, the droplet diameter is $r_{D}=21 \mu \mathrm{m}$ and the recording distance is $z=360.426 \mathrm{~mm}$. For the evaporating droplet, the surrounding refractive index gradient is defined by $\sigma=80 \mu \mathrm{m}$ and $n_{s}=8.5 \times 10^{-5}$.

\section{D. Beam divergence effect for evaporating droplets}

In section 3.A an equivalence has been shown between holograms recorded under plane wave and focused beam illuminations. This equivalence consists of a magnification ratio affecting both the measured droplet radius $r_{D}$ and its distance $z_{E}$ to the sensor. In case of evaporating droplets, the surrounding refractive index gradient is defined by the two other parameters $n_{s}$ and $\sigma$. The equivalence is preserved with the same magnification ratio $G$ applied to $r_{D}, z_{E}$ and to the decay parameter $\sigma$, providing that the refractive index deviation $n_{s}$ at the droplet surface is divided by $G$. This equivalence is illustrated in Fig. 10 where two particle sizes are considered. For $r_{D}=20 \mu \mathrm{m}$, the decay parameter is $\sigma=80 \mu \mathrm{m}$ and for $r_{D}=50 \mu \mathrm{m}, \sigma=200 \mu \mathrm{m}$. In both cases, the refractive index deviation at the droplet surface is $n_{s}=5 \times 10^{-5}$. Figure 10, the droplet holograms recorded at distance $z_{E}=360 \mathrm{~mm}$ for a divergent beam illumination $\left(z_{S}=553.5 \mathrm{~mm}\right)$, perfectly overlaps with the holograms produced at distance $G z_{E}$ (with $G=2.86$ ) by droplets of radius $G r_{D}$, decay parameter $G \sigma$ and refractive index deviation at droplet surface $n_{s} / G$ with a plane wave illumination. This equivalence can be used to reduce computation time as it is smaller for plane wave illumination (LMT) than for focused beam (GLMT). However, computation time increases with particle size and, in the present case, the total gain in computation time using the plane wave equivalence is limited to a factor 4.

\section{Conclusion}

GLMT for multilayered sphere was used to simulate evaporating droplet holograms and to show the effect of refractive index gradient in surrounding medium. Simu-

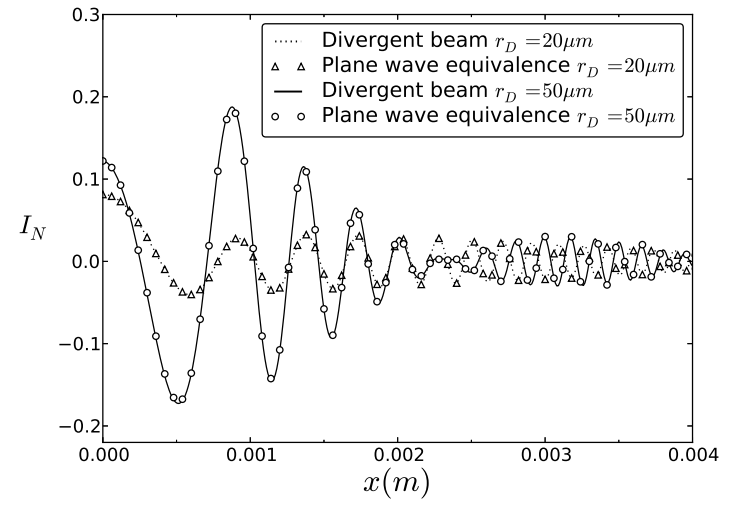

Fig. 10. Comparison between holograms computed for a divergent beam illumination and equivalent holograms computed for a plane wave illumination and magnified droplet parameters.

lations reproduce well the unusual central fringe brightness observed on evaporating Ether droplet holograms and show how a weigthed mask method can be used to reconstruct particle location and size with an 'inverse problems' approach based on a standard parametric hologram formation model. Diverging beam effect is also considered, showing that the equivalence with plane wave illumination can be extended to the case of evaporating droplets. Results presented in this paper open the way to a complete and accurate characterization of evaporating droplets using digital in-line holography together with an 'inverse problems' approach. These approach requires a hologram formation model which takes into account the surrounding medium refractive index gradient. However, GLMT is very time consuming compared to model based on Fresnel propagation integrals, and it is limited to gradients with radial symmetry. To increase the applicative potential of this work, a less time consumming model could be developped. To this purpose, GLMT computations could be used as a reference to validate this model.

\section{Acknowledgments}

This work takes place in the MORIN project (3D Optical Measurements for Research and INdustry), which is supported by the french government through the "Agence Nationale de la Recherche" (ANR) and the "Programme Avenir Lyon-Saint-Etienne" (PAL-SE).

\section{References}

[1] S. Guella, S. Alexandrova, A. Saboni, "Evaporation of a free falling droplet", Int. J. Therm. Sci., 47, 886-898 (2008).

[2] J. S. Wu, Y. J. Liu, H. J. Sheen, "Effect of ambient turbulence and fuel properties on the evaporation rate of single droplets, Int. J. Heat Mass Transfer, 44, 45934603 (2001). 
[3] C. Maqua, G. Castanet, F. Grisch, F. Lemoine, T. Kristyadi, S. Sazhin, "Monodisperse droplet heating and evaporation : experimental study and modelling", Int. J. Heat Mass Transfer, 51, 3932-3945 (2008).

[4] F. Soulez, L. Denis, C. Fournier, E. Thiebaut and C. Goepfert "Inverse-problem approach for particle digital holography: accurate location based on local optimization", J. Opt. Soc. Am. 24, 1164-1171 (2007).

[5] F. Soulez, L. Denis, C. Fournier, E. Thiebaut and C. Goepfert, "Inverse problem approach in particle digital holography: out-of-field particle detection made possible", J. Opt. Soc. Am. 24, 3708-3716 (2007).

[6] S. H. Lee, Y. Roichman, G. R. Yi, S. H. Kim, S. M. Yang, A. van Blaaderen, P. van Oostrum, and D. G. Grier, "Characterizing and tracking single colloidal particles with video holographic microscopy", Optics Express 15 (26), p. 18275-18282 (2007).

[7] D. Chareyron, J.L. Marié, C. Fournier, G. Gire, N. Grosjean, L. Denis, M. Lance and L. Méès, "Testing an in-line digital holography 'inverse method' for the Lagrangian tracking of evaporating droplets in homogeneous nearly isotropic turbulence", New J. of Physics, 14, 043039 (2012).

[8] L. Lorenz, "Lysbevaegelsen i og uden for en af plane Lysbølger belyst Kulge". Vidensk. Selk. Skr., 6, 1-62 (1890).

[9] G. Mie "Beiträge zur Optik Trüber Medien speziell kolloidaler Metallösungen", Ann. der Phys., 330(3), 377445 (1908).

[10] P. Debye, "Der Lichtdruck auf Kugeln von beliebigem Material", Ann. der Phys., 335(11), 57-136 (1909).

[11] G. Gouesbet and G. Gréhan "Generalized Lorenz-Mie theories, from past to future", Atomization and Sprays, 10(3-5), 277-333, (2000).
[12] G. Gouesbet, and G. Gréhan, "Generalized Lorenz-Mie Theories", Springer Berlin Heidelberg (2011).

[13] F. Onofri, G. Gréhan and G. Gouesbet, "Electromagnetic scattering from a multilayered sphere located in an arbitrary beam", Appl. Opt., 34, 7113-7124 (1995).

[14] G. Gouesbet, B. Maheu and G. Gréhan, "The order of approximation in a theory of the scattering of a Gaussian beam by a Mie scatter center", J. Optics (Paris), 16(5), 239-247 (1985).

[15] B. Maheu, G. Gouesbet and G. Gréhan, "A concise presentation of the generalized Lorenz-Mie theory for arbitrary location of the scatterer in an arbitrary incident profile", J. Optics (Paris), 19(2), 59-67 (1988).

[16] G. Gouesbet and J. A. Lock, "Rigorous justification of the localized approximation to the beam-shape coefficients in generalized Lorenz-Mie theory. II. Off-axis beams", J. Opt. Soc. Am. A, 11(9), 2516-2525 (1994).

[17] C. S. Vikram and M. L. Billet, "Some salient features of in-line Fraunhofer holography with divergent beams", Optik 78(2), 80-86 (1988).

[18] D. Lebrun, D. Allano, L. Méès, F. Walle, F., R. Boucheron and D. Fréchou, "Size measurement of bubbles in a cavitation tunnel by digital in-line holography", Appl. Opt., 50(34), H1-H9 (2011).

[19] S. Saengkaew, T.Charinpanitkul, H. Vanisri, W. Tanthapanichakon, Y. Biscos, N. Garcia, G. Lavergne, L. Méès, G. Gouesbet and G. Gréhan, "Rainbow refractometry on particles with radial refractive index gradients", Exp. in Fluids 43(4), 595-601 (2007).

[20] G. R. Toker and J. Stricker, "Holographic study of suspended vaporizing volatile liquid droplets in still air", Int. J. Heat Mass Transfer., 39(16), 3475-3482 (1996).

[21] G. R. Toker and J. Stricker, "Study of suspended vaporizing volatile liquid droplets by an enhanced sensitivity holographic technique : additional results", Int. J. Heat Mass Transfer., 41(16), 2553-2555 (1998). 